\title{
Vibrational properties and magnetic specific heat of the covalent chain antiferromagnet $\mathrm{RbFeSe}_{2}$
}

\author{
A. G. Kiiamov, ${ }^{1,2}$ Y. V. Lysogorskiy, ${ }^{1,3}$ F. G. Vagizov, ${ }^{1}$ L. R. Tagirov, ${ }^{1,4}$ D. A. Tayurskii, ${ }^{1,2}$ Z. Seidov, ${ }^{5,6}$ \\ H.-A. Krug von Nidda, ${ }^{5}$ V. Tsurkan,,${ }^{5,7}$ D. Croitori, ${ }^{7}$ A. Günther, ${ }^{5}$ F. Mayr, ${ }^{5}$ and A. Loidl ${ }^{5}$ \\ ${ }^{1}$ Institute of Physics, Kazan Federal University, Kazan 420008, Russia \\ ${ }^{2}$ Centre for Quantum Technologies, Kazan Federal University, 420008 Kazan, Russia \\ ${ }^{3}$ Atomistic Modelling and Simulation, ICAMS, Ruhr-University Bochum, D-44801 Bochum, Germany \\ ${ }^{4}$ Zavoisky Physical-Technical Institute, FRC Kazan Scientific Center of RAS, 420029 Kazan, Russia \\ ${ }^{5}$ Experimental Physics V, Center for Electronic Correlations and Magnetism, University of Augsburg, D-86135 Augsburg, Germany \\ ${ }^{6}$ Institute of Physics, Azerbaijan National Academy of Sciences, AZ-1143 Baku, Azerbaijan \\ ${ }^{7}$ Institute of Applied Physics, MD-20208 Chisinau, Moldova
}

(Received 3 September 2018; revised manuscript received 9 November 2018; published 10 December 2018)

\begin{abstract}
The magnetic specific heat of $\mathrm{RbFeSe}_{2}$ and the spin state of $\mathrm{Fe}^{3+}$ ions in the compound have been studied. Phonon dispersion and phonon density of states (PDOS), element specific and total, were evaluated from firstprinciples calculations. It is shown that iron atoms in quasi-one-dimensional chains have dramatically different vibrational properties against $\mathrm{Rb}$ and Se atoms: the Fe PDOS is mostly concentrated within two Einstein-like optical phonon peaks at high frequencies. Analysis of our Mössbauer data for $\mathrm{RbFeSe}_{2}$, utilizing the calculated Fe PDOS as well as our optical absorption measurements, have shown full agreement with the location of the high-frequency optical-type lattice vibrations within the $\mathrm{FeSe}_{4}$ tetrahedra. The calculated PDOS was utilized to evaluate the lattice contribution to the specific heat. The phonon heat capacity has been used to evaluate the magnetic specific heat of the quasi-one-dimensional antiferromagnetically correlated $\mathrm{Fe}^{3+}$ ion chains in $\mathrm{RbFeSe}_{2}$. An intermediate spin state $S=3 / 2$ has been found most closely relevant to our magnetic entropy analysis for $\mathrm{Fe}^{3+}$ ions in $\mathrm{RbFeSe}_{2}$.
\end{abstract}

DOI: 10.1103/PhysRevB.98.214411

\section{INTRODUCTION}

The recent discovery of iron-based superconductors [1-4] has triggered enormous interest in iron-pnictide and chalcogenide materials. The crystal structure of these iron-based superconductors contains two -dimensional (2D) layers built of $\mathrm{Fe} \mathrm{Pn}_{4}$ or $\mathrm{FeCh} h_{4}(\mathrm{Pn}=$ pnictogens, $\mathrm{Ch}=$ chalcogens $)$ tetrahedra. Recently [5], pressure-induced superconductivity has been discovered in the spin-ladder compound $\mathrm{BaFe}_{2} \mathrm{~S}_{3}$ consisting of one dimensional (1D) chains of $\mathrm{FeS}_{4}$ tetrahedra. The mechanism of superconductivity and its relation to the crystal structure in these systems of reduced dimensionality are still under debate. In order to achieve deeper insight into the nature of superconductivity and its relation to magnetism, the study of materials containing similar building blocks is of significant interest.

In our previous paper [6] we have studied magnetization, heat capacity, and Mössbauer effect in single crystals of the ternary iron selenide $\mathrm{RbFeSe}_{2}$. This compound belongs to the family $A \mathrm{FeCh} h_{2}(A=$ alkali metal or $\mathrm{Tl})$, which are quasi-1D systems by crystal structure [7-9], although most of them undergo 3D antiferromagnetic order [8-17] with Néel temperatures $T_{\mathrm{N}}$ lying in the range of $188-310 \mathrm{~K}$. Magnetic susceptibility, specific heat, and Mössbauer spectra of $\mathrm{RbFeSe}_{2}$ were measured in a wide range of temperatures and interpreted in terms of conventional approaches. In particular, the temperature dependence of the specific heat $C(T)$ was analyzed approximating the lattice contribution by one Debye and two Einstein vibration modes. The best fit was achieved with the Debye temperature $\theta_{\mathrm{D}}=110 \mathrm{~K}$ and the two Einstein temperatures $\theta_{\mathrm{E} 1}=158 \mathrm{~K}$ and $\theta_{\mathrm{E} 1}=520 \mathrm{~K}$. At the same time, the traditional treatment of the temperature dependence of the Mössbauer spectral area (Lamb-Mössbauer factor $[18,19]) f_{M}(T)$ within the Debye approximation has given $\theta_{\mathrm{MD}}=223 \mathrm{~K}$ (at $T<T_{\mathrm{N}}$ ). This value is twice as large as the Debye temperature $\theta_{\mathrm{D}}=110 \mathrm{~K}$ deduced from the specific heat. It was suggested in Ref. [6] that high-frequency phonons dominate for the iron sites, because $\mathrm{FeSe}_{4}$ tetrahedra are the most rigid units of the $\mathrm{RbFeSe}_{2}$ lattice structure. As the Mössbauer effect is affected only by the iron-nuclei motion, being some kind of local probe of lattice vibrations, their high frequencies come out through the elevated Debye temperature $\theta_{\mathrm{MD}}$ when described in the standard Debye approximation. No quantitative estimations to resolve the contradiction were proposed so far, because of the absence of information on the phonons in highly anisotropic $\mathrm{RbFeSe}_{2}$.

There is another common feature of the specific heat in low-dimensional ternary iron chalcogenides (and, at least, in some oxychalcogenides): the $\lambda$ anomaly at the transition to antiferromagnetic order is very weak if visible at all [20-26]. For $\mathrm{RbFeSe}_{2}$ the magnetic contribution, treated as the difference between the experimentally measured $C(T)$ and the calculated lattice contribution around the $\lambda$ anomaly at $T_{\mathrm{N}}=248 \mathrm{~K}$, yields an entropy change $\Delta S_{M}=0.52 \mathrm{~J} \mathrm{~K}^{-1} \mathrm{~mol}^{-1}$ [6]. This is one order of magnitude smaller than the entropy change $\Delta S_{1 / 2}=R \ln 2=5.76 \mathrm{~J} \mathrm{~K}^{-1} \mathrm{~mol}^{-1}$ theoretically expected for an order-disorder transition of low-spin $S=1 / 2 \mathrm{Fe}^{3+}$ ions. Although it is seen that, because of the equipment limitations 
in temperature $T<300 \mathrm{~K}$, the contribution of higher temperatures to the magnetic specific heat has not been accounted for (see the inset (a) in Fig. 5 of Ref. [6]), the missing area is definitely insufficient to pull up the entropy change by one order of magnitude. However, despite the small entropy change at $T_{\mathrm{N}}$, the measured magnetic moment of $2.66 \mu_{\mathrm{B}}$ per iron ion $[9,10]$ contradicts a low-spin state.

To shed light on the origin of these contradictions, in this paper we perform first-principles calculations of electronic band structure and phonon spectra for $\mathrm{RbFeSe}_{2}$. The bandstructure calculations account for the spin polarization due to magnetic constituents of the crystal structure. This allows us to estimate the magnetic moment located at the iron site. The calculations of the phonon spectra are performed element specifically, i.e., the phonon density of states (PDOS) is calculated for rubidium, iron, and selenium separately. Thus, the contributions of each element of the lattice to the specific heat-or to other quantities which depend on lattice vibrations - can be identified. To corroborate our PDOS calculations we present infrared absorption measurements on single crystals of $\mathrm{RbFeSe}_{2}$ and compare the spectroscopy data with our calculations. We demonstrate that our approach successfully accounts for the above-mentioned controversy between different kinds of measurements.

\section{DENSITY-FUNCTIONAL THEORY CALCULATIONS}

\section{A. Computational background}

The $a b$ initio calculations were performed by means of density-functional theory (DFT) utilizing the Vienna $A b$ initio Simulation Package (VASP 5.3) [27-30]. The electron-ion interactions were taken into account using the projector-augmented wave (PAW) method. The PAW method is a frozen-core one that utilizes the exact shape of the wave functions of the valence electrons instead of pseudo-wave functions [31]. The Perdew-Burke-Ernzerhof generalized gradient approximation (GGA) was applied for the exchange and correlation corrections [32]. The $\mathrm{Rb}\left(4 p^{6} 5 s^{1}\right), \mathrm{Fe}\left(3 d^{6} 4 s^{2}\right)$, Se $\left(4 s^{2} 4 p^{4}\right)$ electrons of the valence shell were treated explicitly, whereas the remaining electrons of the cores were taken into account by using pseudopotentials. The maximum energy for the plane-wave basis set was selected to be equal to $300 \mathrm{eV}$. The $k$-point mesh was a $3 \times 2 \times 3$ Monkhorst-Pack grid which corresponds to the actual spacing of $0.300 \times 0.259$ $\times 0.202$ per $\AA$ [33]. Equilibrium geometry was obtained after several stages of full structural relaxation including atomic positions, cell shape, and cell volume. The PDOS were obtained by means of a direct approach of harmonic approximation making use of the MEDEA-PHONON software [34]. The so-called direct approach to the lattice dynamics is based on the $a b$ initio evaluation of forces on all atoms by a set of finite displacements of a few atoms within an otherwise perfect crystal. The totally optimized equilibrium structure was used for calculation of the phonon dispersion. The lattice parameters obtained after the lattice relaxation are given by $a=7.520 \AA, b=12.153 \AA, c=5.574 \AA$, and the angle $\beta=111.83^{\circ}$. A slight deviation of the calculated lattice parameters from the experimental ones, Ref. [6], of about $1-2 \%$ percent is typical for DFT calculations [35].
TABLE I. Calculated vibrational frequencies of the phonon modes of $\mathrm{RbFeSe}_{2}$. The phonon modes are labeled according to the irreducible representations of the $C_{2}$ point-group symmetry.

\begin{tabular}{lc}
\hline \hline Mode (Mulliken symbol) & Frequency, THz \\
\hline A & 0 \\
B & 0.007 \\
B & 1.205 \\
A & 1.829 \\
A & 1.880 \\
B & 1.985 \\
B & 2.121 \\
B & 2.164 \\
B & 2.343 \\
B & 2.792 \\
A & 2.807 \\
A & 2.824 \\
B & 3.041 \\
A & 3.090 \\
B & 4.556 \\
A & 4.823 \\
A & 6.362 \\
A & 6.782 \\
B & 6.869 \\
B & 7.103 \\
B & 8.507 \\
A & 8.574 \\
B & 8.878 \\
\hline \hline
\end{tabular}

All calculations accounted for the spin polarization due to the antiferromagnetic state of $\mathrm{RbFeSe}_{2}$. The antiferromagnetic spin pattern was set in accordance with the magnetic structure obtained previously by neutron-diffraction data [10]. The best agreement between the calculated $\left[m(\mathrm{Fe})=2.80 \mu_{\mathrm{B}}\right]$ and the experimental $\left(m(\mathrm{Fe})=2.66 \mu_{\mathrm{B}}\right.$ $[9,10])$ values of the magnetic moment per iron ion was obtained for choosing the Hubbard $U$ parameter equal to zero. For this reason, GGA $+U$ approximation was not used in our calculations.

\section{B. Phonon density of states}

This section presents computed phonon frequencies at the center of the Brillouin zone (Table I) and the phonon density of states (Fig. 1) obtained by integration of the phonon dispersion of $\mathrm{RbFeSe}_{2}$. Each vibrational mode is simultaneously Raman and infrared active and belongs to one of the two irreducible representations of the $C_{2}$ point-group symmetry corresponding to the $\mathrm{RbFeSe}_{2}$ structure. It is seen that the PDOS exhibits a complex structure with two distinct frequency ranges where the density achieves maximal values. The vibrational modes of rubidium atoms have relatively low frequencies, so they provide the main contribution to the frequency range 1-3.5 THz (see the top bar chart of Fig. 1). Iron atoms possess the highest vibrational frequencies and constitute a substantial part of the high-frequency range (6.59.5 THz; see the second bar chart from the top of Fig. 1). Selenium atoms show vibrational modes both in low and high-frequency regimes, and exhibit a nonzero density of states in the intermediate frequency range $3.5-6.5 \mathrm{THz}$ (see 


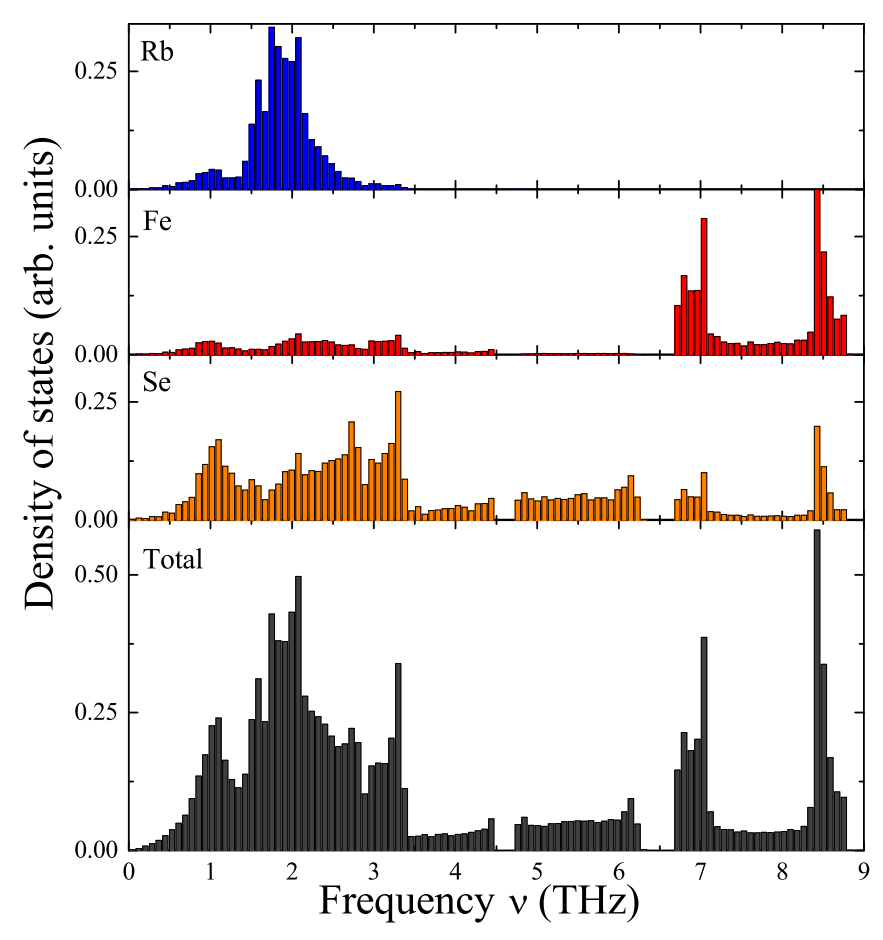

FIG. 1. Calculated phonon density of states as a function of frequency in $\mathrm{RbFeSe}_{2}$ : element specific $(\mathrm{Rb}, \mathrm{Fe}$, and $\mathrm{Se}$ atoms from top toward bottom) and the total PDOS (bottom).

the third bar chart from the top of Fig. 1). The rubidium PDOS resembles a Debye one, and in accordance with the spectra, one could argue that the $\mathrm{Rb}$ atoms vibrate almost independently of the iron atoms in the chain. Such vibrations are expected for weakly bound $\mathrm{Rb}$ atoms.

Given the total PDOS by Fig. 1, the origin of the disagreement in the Debye approximation temperatures, deduced from the specific heat and the Lamb-Mössbauer factor, becomes quite transparent: the total PDOS (the bottom bar chart in Fig. 1), with dominating weight of the low-frequency vibrations, contributes to the specific heat, while the highfrequency vibrations of iron ions (the second bar chart in Fig. 1 from the top) contribute to the Lamb-Mössbauer factor $f_{M}(T)$, yielding a higher Debye temperature as compared to the specific heat.

One more feature of the calculation method needs to be clarified before we move to $C(T)$ and $f_{M}(T)$ calculations: it is known that the DFT approach usually underestimates vibrational frequencies [35,36]. A correction factor is usually established by comparison of calculated and experimental phonon spectra. Known experimental phonon spectra from isostructural systems can be applied, if experimental data for the compound under investigation are unavailable. As an example we refer to the $2 \mathrm{D}$ system $\mathrm{K}_{x} \mathrm{Fe}_{2-y} \mathrm{Se}_{2}$ which is composed of edge-sharing $\mathrm{FeSe}_{4}$ tetrahedra cross-linked by alkali cations [37]. Theoretical phonon frequencies calculated by DFT and experimental phonon spectra of $\mathrm{K}_{1.08} \mathrm{Fe}_{1.6} \mathrm{Se}_{2}$ measured using inelastic neutron-scattering techniques were reported in Ref. [38]. In accordance with those data, the calculated phonon frequencies for the $\mathrm{K}_{0.8} \mathrm{Fe}_{1.6} \mathrm{Se}_{2}$ system are underestimated by about 6-7\%. In our particular case, the frequency-scale renormalization for the PDOS can be verified by analysis of the temperature dependence of the Mössbauer spectral area which is solely determined by vibrations of the iron atoms.

\section{LAMB-MÖSSBAUER FACTOR ANALYSIS}

The temperature dependence of the Mössbauer spectral area for iron ions in $\mathrm{RbFeSe}_{2}$ was presented in Ref. [6]. The iron PDOS (see Fig. 1, the second bar chart from the top) clearly shows that the standard Debye approach, realized in Eq. (2) of Ref. [6], is not applicable to lattice vibrations of the Fe ions, and the fictitious Debye temperatures $\theta_{\mathrm{MD}}=223 \mathrm{~K}$ at temperatures $T<T_{\mathrm{N}}$ and $\theta_{\mathrm{MD}}=194 \mathrm{~K}$ at $T>T_{\mathrm{N}}$ indicate only the relevance of much higher oscillation frequencies for the Lamb-Mössbauer factor as opposed to the specific heat $\left(\theta_{\mathrm{D}}=110 \mathrm{~K}\right.$ for the Debye-mode contribution [6]). To reinterpret the Mössbauer-spectroscopy data we have to return to the initial definition of the Lamb-Mössbauer factor as the temperature dependence of the ratio of recoil-free to total nuclear resonant absorption given by $[18,19]$

$$
f_{M}(T)=\exp \left\{-\frac{\left\langle x^{2}\right\rangle E_{\gamma}^{2}}{(\hbar c)^{2}}\right\},
$$

where $E_{\gamma}$ denotes the gamma-quantum energy, $c$ the velocity of light, and $\bar{h}$ the Planck constant. The mean-square displacement $\left\langle x^{2}\right\rangle$ is the expectation value of the squared vibrational amplitude of the iron ions in the direction of the gamma-radiation propagation. The temperature dependence of the mean-square displacement can be expressed via the phonon density of states [19]:

$$
\left\langle x^{2}\right\rangle=\frac{\hbar}{2 M} \int \frac{1}{\omega} \operatorname{coth}\left(\frac{\hbar \omega}{2 k_{B} T}\right) g_{\mathrm{Fe}}(\omega) d \omega,
$$

where $\omega$ is the phonon frequency, $g_{\mathrm{Fe}}(\omega)$ is the PDOS of Mössbauer nuclei, $M$ is the (effective) nucleus mass, and $k_{B}$ is the Boltzmann constant. Then, instead of taking the traditional next step of transition to the Debye PDOS in $g_{\mathrm{Fe}}(\omega)$, we calculate the temperature dependence of the Mössbauer spectral area, Eq. (1), numerically integrating in Eq. (2) with the partial phonon density of states of iron $g_{\mathrm{Fe}}(\omega)$, given on Fig. 1.

Figure 2 shows the result of the fitting to the experimental temperature dependence of the Mössbauer spectral area. The oscillations of iron ions along the $X, Y$, and $Z$ axes have been accounted for in the analysis without weighting, because $\mathrm{x}$-ray diffraction measurements did not show any significant texture of the samples. The bare iron atom mass has been taken for $M$. The only fitting parameter was the phonon frequency underestimation factor, discussed above, i.e., the scale-correction factor for the frequency. The best agreement with the experiment has been achieved by expanding the frequency scale by a factor of 1.08 .

\section{INFRARED SPECTROSCOPY}

\section{A. Experimental details}

High-frequency phonons are Raman and optically active according to calculations, so infrared (IR) spectroscopy could also clarify the presence of high-frequency vibrations within 


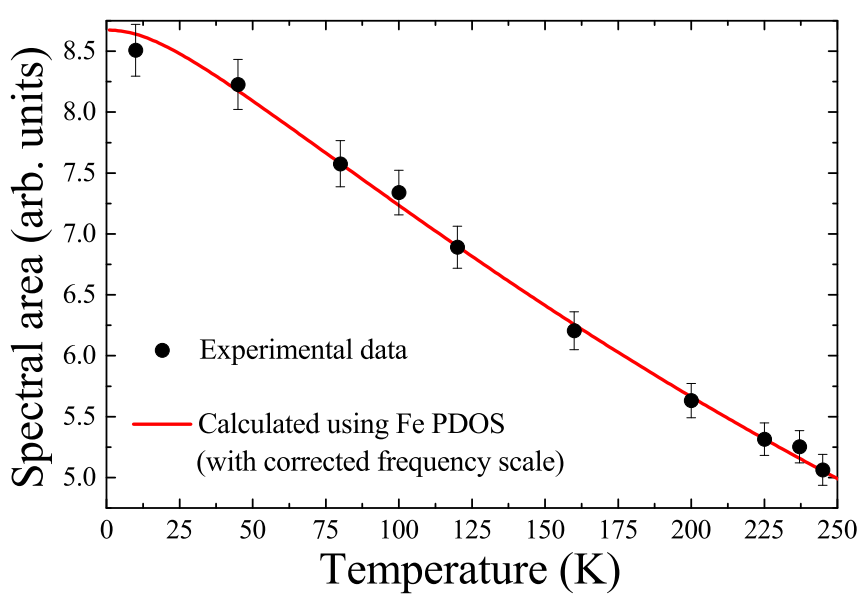

FIG. 2. Temperature dependence of the Mössbauer spectral area of $\mathrm{RbFeSe}_{2}$ : black solid symbols, experiment; red solid line, calculation utilizing the iron partial PDOS.

$\mathrm{FeSe}_{4}$ tetrahedra manifested as singularities (peaks) in the PDOS at $\sim 7.0$ and $\sim 8.5 \mathrm{THz}$ (see Fig. 1 ). The IR absorption was obtained in the wave-number range $100-600 \mathrm{~cm}^{-1}$, which corresponds to the frequency range 3-18 $\mathrm{THz}$, by using a Bruker IFS $113 \mathrm{v}$ spectrometer. The ground sample was dispersed in CsI powder in the ratio $1.35 \mathrm{mg} \mathrm{RbFeSe}$ to $100 \mathrm{mg}$ CsI. This mixture was cold pressed under vacuum to obtain a disklike pellet with 13-mm diameter.

\section{B. Infrared absorption spectrum}

The experimental infrared absorption spectrum shows two broad lines, which are compared to the frequency-scale corrected PDOS in Fig. 3. It is clearly seen that the resonance frequencies of the two experimental absorption peaks fairly fit to the lower bounds of the frequency ranges 7.30-7.70 and 9.00-9.50 THz corresponding to the oscillation modes of iron

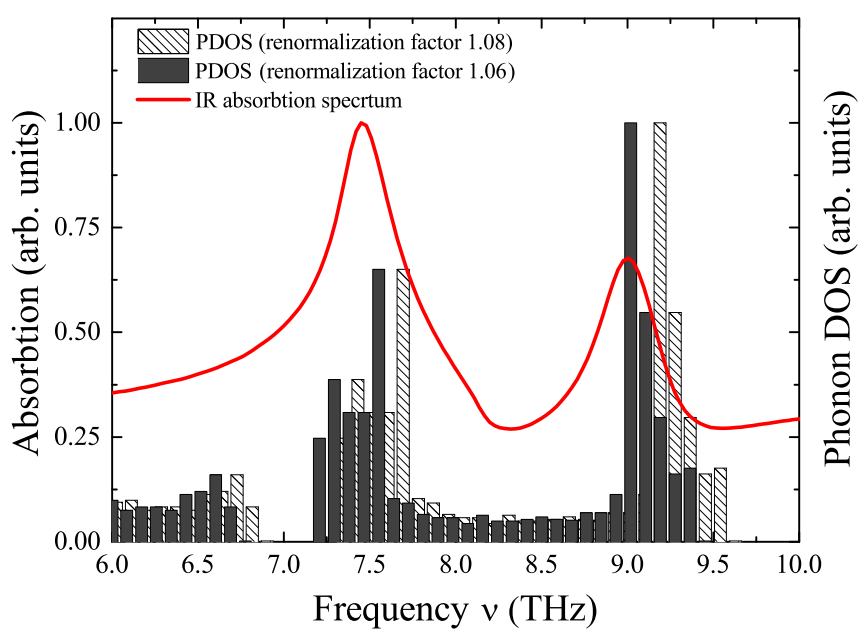

FIG. 3. Infrared absorption spectrum of $\mathrm{RbFeSe}_{2}$ compared with the calculated PDOS of $\mathrm{RbFeSe}_{2}$. The frequency scale of the PDOS is corrected in accordance with the Mössbauer data (see details in Sec. III) and to get best coincidence of the peaks of PDOS maxima with the IR absorption maxima (solid bar charts). and selenium atoms scaled by a factor of 1.08 according to the Mössbauer results (dashed bar chart). One can argue that the experimental IR-absorption spectrum qualitatively confirms the calculated PDOS, but the frequency scales, as expected, do not exactly match. The IR spectrum has best agreement with the calculated PDOS in case of a scaling factor of 1.06 (solid bar chart). A $2 \%$ difference in the scaling factors from Mössbauer and optical experiments can be qualitatively explained by possible slight structural distortion because of the surface oxidation upon IR measurements and a corresponding phonon-frequencies shift. As the IR measurement was performed in ambient air atmosphere, and because of the optical frequency domain, we expect it to be more sensitive to surface modifications than the Mössbauer measurements, which were conducted in argon protective atmosphere, both the absorber preparation and the measurements, and moreover, because gamma rays refer to ionizing radiation, one expects vanishing influence of the surface properties on the measurement results.

The intermediate conclusion is that the $a b$ initio calculated PDOS well describes the temperature dependence of the Lamb-Mössbauer factor and agrees with the experimental infrared absorption spectrum. For further quantitative analysis of the thermodynamic properties we use the frequency-scaling factor 1.08 .

\section{SPECIFIC HEAT ANALYSIS}

We assume that the total specific heat originates from two contributions, a lattice specific heat due to acoustic and optical phonons, and a magnetic one determined by thermal population of excited magnetic states. We estimate the magnetic specific heat as the difference between the total experimental heat capacity and the calculated vibrational part. The PDOS enables us to calculate directly the lattice contribution to the specific heat by using the harmonic approximation [39]. In the harmonic approximation the lattice heat capacity can be determined as follows [40]:

$$
C_{V}(T)=D N k_{B} \int\left[\frac{\hbar \omega / 2 k_{B} T}{\sinh \left(\hbar \omega / 2 k_{B} T\right)}\right]^{2} g_{T}(\omega) d \omega,
$$

where $D$ is the number of degrees of freedom in the unit cell (3 in our case), and $g_{T}(\omega)$ is the total PDOS (Fig. 1, bottom panel).

In most of the cited experimental works [20-26] the temperature dependence of the specific heat at constant pressure, $C_{P}(T)$, is presented, because the specific heat is usually measured at ambient conditions in a wide range of temperatures so that the thermal expansion could not be neglected. Since, from general thermodynamic approach, $C_{P}(T)-C_{V}(T)=$ $-T\left[(\partial V / \partial T)_{P}\right]^{2} /(\partial V / \partial P)_{T}>0$ (see Ref. [41], Sec. 16), therefore $C_{P}(T)$ is always larger than $C_{V}(T)$ approaching the latter at low temperatures. For solids (Ref. [41], Sec. 67),

$$
C_{P}(T)-C_{V}(T)=-T \frac{\alpha^{2} V_{0}^{2}}{d V_{0} / d P}=\alpha^{2} B V_{0} T,
$$

where $\alpha(P)$ denotes the thermal expansion coefficient, $V_{0}$ the molar volume, and $B=-V_{0} /\left(d V_{0} / d P\right)$ is the bulk modulus. 


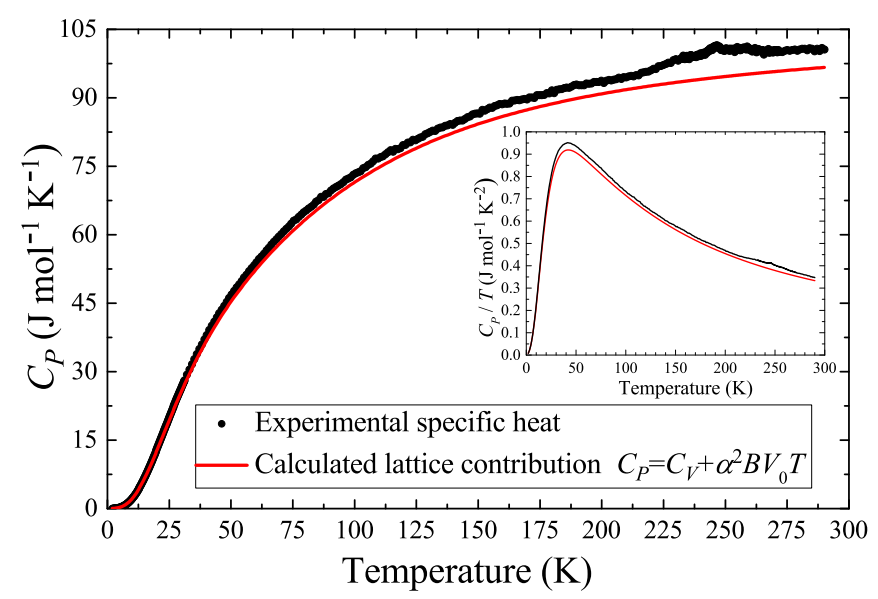

FIG. 4. Temperature dependence of the specific heat $C_{P}$ of $\mathrm{RbFeSe}_{2}$ : black points, experimental data; red line, calculated lattice contribution to the specific heat at constant pressure $C_{P}=C_{V}+$ $0.008\left[\mathrm{~J} / \mathrm{mol} \mathrm{K}^{2}\right] T$ (see details in Sec. V). Inset: the experimental and calculated specific heat drawn as $C_{P}(T) / T$.

In the present study, the bulk modulus of $\mathrm{RbFeSe}_{2}$ has been estimated directly from the second derivative of the total energy as a function of the unit-cell volume by $a b$ initio calculations: $B=17.10 \mathrm{GPa}$. The total energy per unit-cell volume itself was obtained from a polynomial fit, which was truncated after the fourth-order term. The thermal expansion coefficient $\alpha$ was estimated utilizing the crystal-structure data presented for room temperature and $14 \mathrm{~K}$ in Ref. [10], so that the specific heat at constant pressure could be expressed as $C_{P} \approx C_{V}+0.008\left[\mathrm{~J} / \mathrm{mol} \mathrm{K}^{2}\right] T$. This expression was used for the calculated specific heat shown in Fig. 4 (see the legend).

Figure 4 compares the calculated lattice contribution to the experimentally obtained total specific heat. It is seen that below $12 \mathrm{~K}$ the calculated lattice contribution demonstrates a cubic dependence on temperature (the inset in Fig. 4), whereas the experimental specific heat deviates from this dependence. This difference is associated with the magnetic specific heat that is obviously expected for the case of the quasi-onedimensional antiferromagnetically ordered $\mathrm{RbFeSe}_{2}$.

The magnetic contribution to the specific heat $C_{m}(T)$ is shown in Fig. 5. The corresponding magnetic entropy change can be calculated from the experimentally measured magnetic specific heat as $\Delta S_{M}=\int\left[C_{m}(T) / T\right] d T$. The integration from zero temperature to $290 \mathrm{~K}$ gives $\Delta S_{M}=$ $6.03 \mathrm{~J} \mathrm{~K}^{-1} \mathrm{~mol}^{-1}$, which is an estimation of the lower boundary, because of the temperature limit $(T<290 \mathrm{~K})$ of our equipment. For quasi-one-dimensional systems one expects magnetic fluctuations to persist far above the Néel temperature. Indeed, Figs. 6 to 9 in Ref. [42] for purely 1D spin chain with antiferromagnetic exchange and Figs. 1 and 6 in Ref. [43] for 1D to 3D antiferromagnets clearly show a large enhancement of the high-temperature tail of the specific heat when lowering the lattice dimensionality. In fact, our $\mathrm{RbFeSe}_{2}$ compound is a quasi-1D, highly anisotropic antiferromagnet with the 3D Néel temperature $\mathrm{TN}=248 \mathrm{~K}$ induced by weak interchain interaction (exchange and/or dipole-dipole). The absence of the magnetic susceptibility maximum $[42,44]$ in the temperature range from the Néel temperature until $720 \mathrm{~K}$

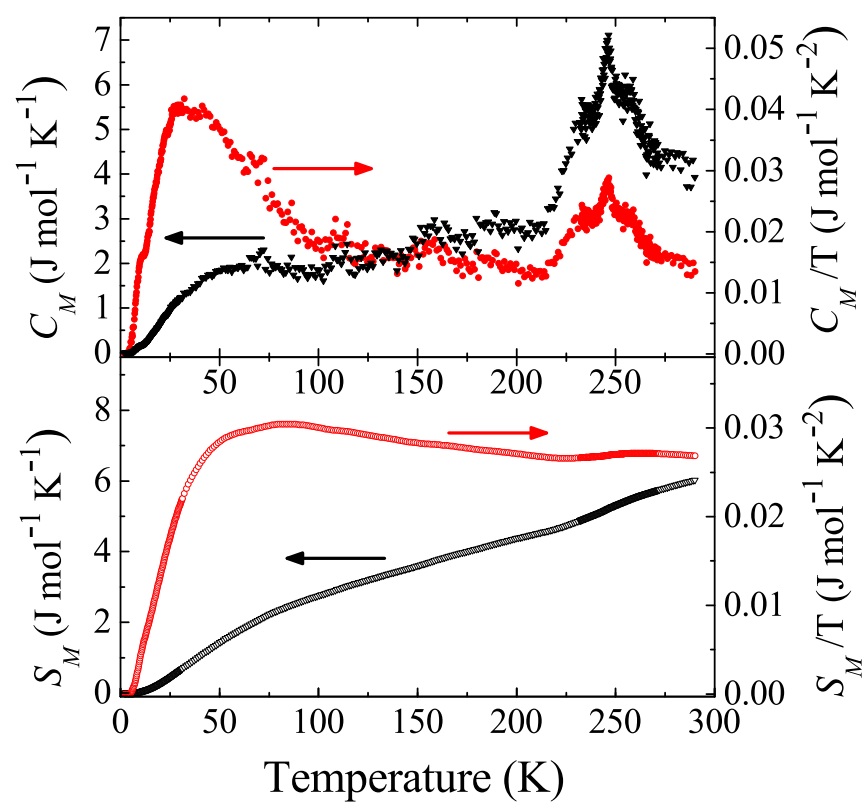

FIG. 5. Temperature dependence of the magnetic heat capacity $C_{M}(T)$ (top panel, black solid triangles) obtained as the difference between the experimentally measured specific heat and the calculated lattice contribution. Red solid circles, top panel, display the temperature dependence of $C_{M}(T) / T$. Bottom panel, black open triangles display change in the magnetic entropy with temperature, $S_{M}(T)$ $\left(C_{M}(T) / T\right.$ integrated from zero temperature up to the current value of the temperature); red open circles display temperature dependence of $S_{M}(T) / T$.

(see Fig. 3 in Ref. [6]) indicates that the intrachain exchange in temperature units is larger than the highest temperature of the measurements. This, in turn, means that 1D fluctuations and the enhanced specific heat might still persist up to $700 \mathrm{~K}$, above which the sample begins to thermally deteriorate. Therefore, in spite of the $1 / T$ factor in the integrand of the magnetic entropy expression given above, there is plenty of room for fluctuations above $T_{\mathrm{N}}$ to contribute to the entropy change upward from $6.03 \mathrm{~J} \mathrm{~K}^{-1} \mathrm{~mol}^{-1}$ received in the temperature range $0-290 \mathrm{~K}$. This has to be compared with the theoretical expectation for the possible cases of low-spin $1 / 2$, intermediate-spin $3 / 2$, and high-spin $5 / 2$ for $\mathrm{Fe}^{3+}\left(3 d^{5}\right)$ atoms in $\mathrm{RbFeSe}_{2}$, where the corresponding entropy change $\Delta S_{M}$ at the antiferromagnet-paramagnet orderdisorder transition is given by $R \ln 2=5.76 \mathrm{~J} \mathrm{~K}^{-1} \mathrm{~mol}^{-1}$, $R \ln 4=11.52 \mathrm{~J} \mathrm{~K}^{-1} \mathrm{~mol}^{-1}$, and $R \ln 6=14.89 \mathrm{~J} \mathrm{~K}^{-1} \mathrm{~mol}^{-1}$, respectively. Thus, the experimentally obtained value of the magnetic entropy change $\Delta S_{M}$ comes closer to that for the intermediate-spin state $S=3 / 2$. Note that also the reported magnitudes of hyperfine fields on iron nuclei $(\sim 216 \mathrm{kOe}$ at liquid-helium temperature [6]) and of the magnetic moment at the iron ions $\left(\sim 2.66 \mu_{B}[9,10]\right)$ are not typical either for the $S=1 / 2$, or for the $S=5 / 2$ spin state. Therefore, the set of data for $\Delta S_{M}$, hyperfine field, and magnetic moment favors the intermediate-spin state $S=3 / 2$ of the iron atoms in $\mathrm{RbFeSe}_{2}$. The splitting of the $\mathrm{Fe}^{3+} d$ orbitals in the case of edge-sharing $\mathrm{FeSe}_{4}$ tetrahedra in $\mathrm{RbFeSe}_{2}$ was already reported in Ref. [10]: the $S=3 / 2$ intermediate-spin state can arise from the distortion of the tetrahedra and the 
corresponding splitting of orbital triplet and doublet by the associated low-symmetry crystal field. The delocalization of some fraction of the iron $d$ electrons in $\mathrm{RbFeSe}_{2}$ leads to reduced values of the hyperfine field and of the magnetic moment with respect to those typical for the $S=3 / 2$ case $\left(\sim 330 \mathrm{kOe}\right.$ and $\sim 3 \mu_{B}$, respectively, reported for ionic oxides, Ref. [18], Chap. 7).

Now we will discuss the temperature dependence of the specific heat in more detail. Since below $T_{\mathrm{N}}=248 \mathrm{~K}$ $\mathrm{RbFeSe}_{2}$ is a 3D antiferromagnet, one has to expect a $T^{3}$ contribution to $C_{m}(T)$ from antiferromagnetic magnons [45] which is hardly distinguished from the lattice contribution, if the Debye-like low-frequency part of the PDOS (see Fig. 1, a region adjoining a frequency of $1 \mathrm{THz}$ ) is characterized by a Debye temperature $\theta_{\mathrm{D}}$ several times smaller than $T_{\mathrm{N}}$ which seems to be our case. Indeed, the inset in Fig. 4 does not show the additional contribution of $T^{3}$ functional dependence; instead, a tiny upward deviation of the experimentally measured $C_{P}(T)$ (black line) from the low-temperature $T^{3}$ dependence (red line) indicates an additional source of heat capacity with stronger dependence on $T$ than $T^{3}$.

Much more significant magnetic contribution to the specific heat $C_{m}(T)$ is seen in Fig. 5 as a broad "feature" in the temperature range 30-200 K. Indeed, because of the quasione-dimensional structure of $\mathrm{RbFeSe}_{2}$, one could expect that quantum fluctuations, which otherwise prevent magnetic ordering in ideal 1D spin chains according to the MerminWagner theorem $[46,47]$, persist well below the 3D ordering temperature $T_{\mathrm{N}}=248 \mathrm{~K}$. Numerous theoretical and experimental studies (see, for example, Refs. [42-44,48-50] and references therein) have reported a nearly linear temperature dependence of the magnetic specific heat for Heisenberg[spin-1/2 to 3] or $X Y$ - [spin-1/2] antiferromagnetic chains at temperatures below the nearest-neighbor exchange energy in temperature units (see a collection of the results in Figs. 6, 15, 18, and Table I of Ref. [43]). As far as our system is quasi-one-dimensional, it undergoes a transition into a 3D antiferromagnetic state with a Néel temperature roughly equal to the intrachain mean-field $T_{\mathrm{N}}$ (MF-intra) times an interchain to intrachain exchange integral ratio. The latter is much smaller than unity for highly anisotropic systems, which is why the intrachain exchange energy is expected to be much larger than the 3D antiferromagnetic transition temperature (a cusp at $T_{\mathrm{N}}=248 \mathrm{~K}$ in Fig. 5). Under these conditions the temperature range of our measurements undoubtedly falls into the range of quasilinear $C_{m}(T)$ predicted by the theories for Heisenberg antiferromagnetic chains.

In Refs. [51-57] it was shown that in a uniform external magnetic field $H_{0}$ a field-induced gap develops because of induced exchange anisotropy due to transverse staggered magnetic field $h \ll H_{0}$ and Dzyaloshinsky-Moriya [58] interaction. Then, at low temperatures the specific heat is suppressed by the gap; on the contrary, above the gap the specific heat is enhanced since the total number of degrees of freedom is conserved. At higher temperatures $C_{m}(T)$ recovers the behavior for the uniform chain. This temperature dependence resembles the one shown in Fig. 5; however, it is unlikely that the above-mentioned model can be directly applied to our case: The theoretical model is developed for spin- $1 / 2$ chains, and there was no uniform magnetic field $H_{0}$ applied upon our $C_{P}(T)$ measurements. The crucial difference is that our quasi-one-dimensional system undergoes a $3 \mathrm{D}$ antiferromagnetic transition at zero external magnetic field that implies the existence of a strong local staggered interchain molecular field transverse to the chain direction [10]. The role of interchain interaction in the temperature behavior of the specific heat was analyzed in Refs. [59-62] for the quasi-one-dimensional spin-1/2 chain compound $\mathrm{Yb}_{4} \mathrm{As}_{3}$. To our best knowledge, there is no appropriate theory to treat our particular case of magnetic specific heat in quasi-one-dimensional spin-3/2 chains with the interchain interaction triggering the transition to the $3 \mathrm{D}$ antiferromagnetic state at $T_{\mathrm{N}}=248 \mathrm{~K}$.

\section{CONCLUSIONS}

We have performed element-specific ab initio calculations of the vibrational properties of the covalent antiferromagnetic chain compound $\mathrm{RbFeSe}_{2}$ and discussed its implications to results from Mössbauer and infrared spectroscopy as well as specific-heat measurements. The calculation revealed that rubidium vibrations are located in the low-frequency domain $1-3.5 \mathrm{THz}$ and represented by dominating acoustic oscillations. On the contrary, iron ion vibrations are high-frequency and predominantly optical-type oscillations in the 6.59.5- THz frequency domain. In the selenium phonon spectrum the low and high frequencies are present as well as oscillations in the intermediate frequency range 3.5-6.5 THz. Using our experimental Mössbauer and infrared data we adjusted the frequency scale of the theoretical phonon density of states. This allowed us to simulate a realistic lattice contribution to the specific heat. The magnetic specific heat was obtained as the difference between the measured total specific heat and the calculated lattice contribution. The entropy change in the temperature interval from 0 to $290 \mathrm{~K}$, associated with this magnetic contribution, amounts to $6.03 \mathrm{~J} \mathrm{~K}^{-1} \mathrm{~mol}^{-1}$ and visually exhibits an extended high-temperature tail above $290 \mathrm{~K}$ due to the quasi-one-dimensionality of the system. This suggests the intermediate $S=3 / 2$ spin state to be realized for the iron ions in the chains. The measured magnetic moment of $2.66 \mu_{\mathrm{B}}[9,10]$ and the calculated moment of $2.80 \mu_{\mathrm{B}}$ per iron ion are in agreement with the intermediate spin state. Our results suggest that the features of the phonon spectrum and thermal properties are general for the whole family of the covalent chain ternary iron chalcogenides of the structure $X \mathrm{Fe} Y_{2}$.

\section{ACKNOWLEDGMENTS}

The authors thank Dana Vieweg for x-ray measurements. This work was partially supported partly by the Deutsche Forschungsgemeinschaft (DFG) within the Transregional Collaborative Research Center TRR 80 "From Electronic Correlations to Functionality" (Augsburg-MunichStuttgart). L.R.T. acknowledges funding by the state assignment No. AAAA-A18-118030690040-8. The work of A.G.K. and D.A.T. was funded by the subsidy allocated to Kazan Federal University for the state assignment in the sphere of scientific activities (Project No. 9779.2017/8.9). 
[1] Y. Kamihara, T. Watanabe, M. Hirano, and H. Hosono, J. Am. Chem. Soc. 130, 3296 (2008).

[2] G. Stewart, Rev. Mod. Phys. 83, 1589 (2011).

[3] E. Dagotto, Rev. Mod. Phys. 85, 849 (2013).

[4] M. Fang, H. Wang, C. Dong, and Q. Huang, J. Phys.: Conf. Ser. 449, 012015 (2013).

[5] H. Takahashi, A. Sugimoto, Y. Nambu, T. Yamauchi, Y. Hirata, T. Kawakami, M. Avdeev, K. Matsubayashi, F. Du, C. Kawashima, H. Soeda, S. Nakano, Y. Uwatok, Y. Ueda, T.Sato, and K. Ohgushi, Nat. Mater. 14, 1008 (2015).

[6] Z. Seidov, H.-A. Krug von Nidda, V. Tsurkan, I. G. Filipova, A. Günther, T. P. Gavrilova, F. G. Vagizov, A. G. Kiiamov, L. R. Tagirov, and A. Loidl, Phys. Rev. B 94, 134414 (2016).

[7] M. R. Harrison and M. G. Francesconi, Coord. Chem. Rev. 255, 451 (2011).

[8] W. Bronger, Angew. Chem. 20, 52 (1981).

[9] W. Bronger and P. Müller, J. Alloys Compd. 246, 27 (1997).

[10] W. Bronger, A. Kyas, and P. Müller, J. Solid State Chem. 70, 262 (1987).

[11] D. Welz, P. Deppe, W. Schaefer, H. Sabrowsky, and M. Rosenberg, J. Phys. Chem. Solids 50, 297 (1989).

[12] Z. Seidov, H.-A. Krug von Nidda, J. Hemberger, A. Loidl, G. Sultanov, E. Kerimova, and A. Panfilov, Phys. Rev. B 65, 014433 (2001).

[13] E. B. Asgerov, N. T. Dang, A. I. Beskrovnyy, A. I. Madadzada, D. I. Ismayilov, R. N. Mehdiyeva, S. H. Jabarov, and E. M. Karimova Semiconductors 49, 879 (2015).

[14] M. Nishi and Y. Ito, Solid State Commun. 30, 571 (1979).

[15] D. Welz, M Kohgi, Y. Endoh, M. Nishi, and M. Arai, Phys. Rev. B 45, 12319 (1992).

[16] D. Welz, S. Itoh, and A. D. Taylor, Europhys. Lett. 34, 293 (1996).

[17] D. Welz and M. Nishi, Phys. Rev. B 45, 9806 (1992).

[18] G. K. Wertheim, Mössbauer Effect: Principles and Applications (Academic, New York, 1964), Chap. 4.

[19] Ph. Gütlich, E. Bill, and A. X. Trautwein. Mössbauer Spectroscopy and Transition Metal Chemistry. Fundamentals and Applications (Springer-Verlag, Berlin, 2011), Chap. 2.

[20] D. C. Johnston, S. C. Mraw, and A. J. Jacobson, Solid State Commun. 44, 255 (1982).

[21] M. Aldzhanov, N. Guseinov, G. Sultanov, and M. Nadzafzade, Phys. Status Solidi B 159, K107 (1990).

[22] J. B. He, D. M. Wang, H. L. Shi, H. X. Yang, J. Q. Li, and G. F. Chen, Phys. Rev. B 84, 205212 (2011).

[23] H. Lei, H. Ryu, A. I. Frenkel, and C. Petrovic, Phys. Rev. B 84, 214511 (2011).

[24] Y. Nambu, K. Ohgushi, Sh. Suzuki, F. Du, M. Avdeev, Y. Uwatoko, K. Munakata, H. Fukazawa, S. Chi, Y. Ueda, and T. J. Sato, Phys. Rev. B 85, 064413 (2012).

[25] H. Lei, H. Ryu, V. Ivanovski, J. B. Warren, A. I. Frenkel, B. Cekic, W.-G. Yin, and C. Petrovic, Phys. Rev. B 86, 195133 (2012).

[26] F. Du, K. Ohgushi, Y. Nambu, T. Kawakami, M. Avdeev, Y. Hirata, Y. Watanabe, T. J. Sato, and Y. Ueda, Phys. Rev. B 85, 214436 (2012).

[27] G. Kresse and J. Hafner, Phys. Rev. B 47, 558 (1993).

[28] G. Kresse and J. Hafner, Phys. Rev. B 49, 14251 (1994).

[29] G. Kresse and J. Furthmuller, Comput. Mater. Sci. 6, 15 (1996).
[30] G. Kresse and J. Furthmuller, Phys. Rev. B 54, 11169 (1996).

[31] P. E. Blöchl, Phys. Rev. B 50, 17953 (1994).

[32] J. P. Perdew, K. Burke, and M. Ernzerhof, Phys. Rev. Lett. 77, 3865 (1996).

[33] H. J. Monkhorst and J. D. Pack, Phys. Rev. B 13, 5188 (1976).

[34] K. Parlinski, Z. Q. Li, and Y. Kawazoe, Phys. Rev. Lett. 78, 4063 (1997).

[35] Y. V. Lysogorskiy, R. M. Eremina, T. P. Gavrilova, O. V. Nedopekin, D. A. Tayurskii, JETP Lett. 100, 652 (2015).

[36] A. Dal Corso, J. Phys: Condens. Matter 25, 145401 (2013).

[37] J. Guo, S. Jin, G. Wang, S. Wang, K. Zhu, T. Zhou, M. He, and X. Chen, Phys. Rev. B 82, 180520 (2010).

[38] R. Mittal, M. K. Gupta, S. L. Chaplot, M. Zbiri, S. Rols, H. Schober, Y. Su, Th. Brueckel, and T. Wolf, Phys. Rev. B 87, 184502 (2013).

[39] C. Kittel, Introduction to Solid State Physics, 7th ed. (Wiley, New York, 1996), Chaps. 4 and 5.

[40] J. M. Ziman, Electrons and Phonons, the International Series of Monographs on Physics (Clarendon, Oxford, 1960), Chap. 1.

[41] L. D. Landau and E. M. Lifshitz, Statistical Physics, 3rd ed., Part 1 (Course of Theoretical Physics, Vol. 5), revised and enlarged by E. M. Lifshitz and L. P. Pitaevsky (Pergamon, New York, 1980).

[42] J. C. Bonner and M. E. Fisher, Phys. Rev. 135, A640 (1964).

[43] L. J. de Jongh and A. R. Miedema, Adv. Phys. 23, 1 (1974).

[44] S. K. Tiwary and S. Vasudevan, Phys. Rev. B 56, 7812 (1997).

[45] J. J. Quinn and K.-S. Yi. Solid State Physics. Principles and Modern Applications (Springer-Verlag, Berlin, 2009), Sec. 10.7 .

[46] N. D. Mermin and H. Wagner, Phys. Rev. Lett. 17, 1133 (1966).

[47] A. Gelfert and W. Nolting, J. Phys.: Condens. Matter 13, R505 (2001).

[48] D. W. Hone and P. M. Richards, Annu. Rev. Mater. Sci. 4, 337 (1974).

[49] J. C. Bonner, J. Appl. Phys. 49, R1299 (1978).

[50] Physics in One Dimension, in Proceedings of an International Conference Fribourg, Switzerland, August 25-29, 1980, Springer Series in Solid-State Sciences Vol. 23, edited by I. Bernasconi and T. Schneider (Springer-Verlag, Berlin, 1981), Part III: Magnetic Chains.

[51] D. C. Dender, D. Davidović, D. H. Reich, C. Broholm, K. Lefmann, and G. Aeppli, Phys. Rev. B 53, 2583 (1996).

[52] D. C. Dender, P. R. Hammar, D. H. Reich, C. Broholm, and G. Aeppli, Phys. Rev. Lett. 79, 1750 (1997).

[53] M. Oshikawa and I. Affleck, Phys. Rev. Lett. 79, 2883 (1997).

[54] I. Affleck and M. Oshikawa, Phys. Rev. B 60, 1038 (1999).

[55] F. H. L. Essler and A. M. Tsvelik, Phys. Rev. B 57, 10592 (1998).

[56] F. H. L. Essler, Phys. Rev. B 59, 14376 (1999).

[57] C. Broholm, G. Aeppli, Y. Chen, D. C. Dender, M. Enderle, P. R. Hamraar, Z. Honda, K. Katsumata, C. P. Landee, M. Oshikawa, L. P. Regnault, D. H. Reich, S. M. Shapiro, M. Sieling, M. B. Stone, M. M. Turnbull, I. Zaliznyak, and A. Zheludev, Magnetized states of quantum spin chains, in High Magnetic Fields, Applications in Condensed Matter Physics and Spectroscopy, Springer Lecture Notes in Physics, Vol. 595, edited by C. Berthier, L. P. Levy, and G. Martinez (SpringerVerlag, Berlin, 2002), p. 211-234.

[58] I. Dzyaloshinskii, J. Phys. Chem. Solids 4, 241 (1958); T. Moriya, Phys. Rev. 120, 91 (1960). 
[59] B. Schmidt, P. Thalmeier, and P. Fulde, Europhys. Lett. 35, 109 (1996).

[60] M. Köppen, M. Lang, R. Helfrich, F. Steglich, P. Thalmeier, B. Schmidt, B. Wand, D. Pankert, H. Benner, H. Aoki, and A. Ochiai, Phys. Rev. Lett. 82, 4548 (1999).
[61] F. Steglich, M. Köppen, P. Gegenwart, T. Cichorek, B. Wand, M. Lang, P. Thalmeier, and B. Schmidt, Acta Phys. Pol. A97, 91 (2000).

[62] B. Schmidt, H. Aoki, T. Cichorek, P. Gegenwart, A. Ochiai, and F. Steglich, J. Phys. Soc. Jpn. 71S, 64 (2002). 